\title{
FUENTES PARA EL ESTUDIO DE LA EMIGRACIÓN DE ALICANTINOS A ARGELIA DURANTE LA ETAPA COLONIAL (1)
}

Por

JOSÉ FERMÍN BONMATI ANTÓN

Importancia de la corriente en la provincia de Alicante

La emigración al Norte de África (fundamentalmente a Argelia francesa) fue, durante gran parte del siglo $X I X$ y primer tercio del siglo $X X$, la corriente casi exclusiva en el ámbito de la provincia de Alicante. En las estadisticas oficiales se refleja claramente este hecho.

CUADRO I Emigrantes alicantinos según lugares de destino. 1885-1895

\begin{tabular}{cccccccc} 
AÑOS & ARGELIA & OTROS AFR. & EUROPA & COLONIAS (1) & AMÉRICA (2) & TOTAL \\
\cline { 1 - 3 } 1885 & 4.460 & 1 & - & - & & 38 & $4.524(3)$ \\
1886 & 8.852 & 0 & - & - & 18 & $8.899(3)$ \\
1887 & 6.465 & 1 & - & - & 23 & $6.642(3)$ \\
1888 & 4.805 & 0 & - & - & 91 & $4.969(3)$ \\
1889 & 6.558 & 3 & - & - & 815 & $7.457(3)$ \\
1890 & 5.125 & 2 & - & - & 133 & $5.328(3)$ \\
1891 & 6.046 & 0 & 47 & 27 & 69 & 6.189 \\
1892 & 5.607 & 2 & 33 & 39 & 43 & 5.724 \\
1893 & 5.511 & 0 & 36 & 42 & 79 & 5.668 \\
1894 & 6.986 & 5 & 114 & 81 & 61 & 7.247 \\
1895 & 5.009 & 19 & 27 & 34 & 140 & 5.229
\end{tabular}

(1) Se trata de las colonias españolas, incluidas las americanas.

(2) No se incluyen las colonias españolas.

(3) No constan los datos específicos de las salidas hacia los países europeos ni hacia las colonias españolas en los años 1885 a 1890.

FUENTE: DIRECCION GENERAL DEL INSTITUTO GEOGRÁFICO Y ESTADISTICO. Estadística de la emigración e inmigración de España, volümenes correspondientes a los periodos $1882-1890$ y $1891-1895$.

(1) Este articulo resume un capítulo de la Memoria de Licenciatura del autor 
El cuadro anterior refleja únicamente la situación de 10 años, a fines del siglo XIX. Cabe preguntarse si lo que expresan estas cifras es un fenómeno meramente coyuntural. No podemos conocer la importancia cuantitativa de la corriente durante épocas anteriores por cuanto las estadísticas españolas sobre emigración arrancan del año 1882, pero múltiples testimonios, a lo largo del siglo $X \mid X$, señalan la gran importancia cualitativa de dicha corriente de emigración a territorio argelino en nuestra provincia, hasta el punto de llegar a identificar «emigración a Argelia» con «emigración en general» de los alicantinos.

Por ejemplo, en 1882, la Comisión Especial para estudiar los medios de contener en lo posible la emigración en España, organismo oficial creado por el Gobierno Sagasta en 1881 para el mejor conocimiento del fenómeno de la emigración en nuestro país, dirigió un interrogatorio con preguntas sobre distintos aspectos de la emigración provincial a las Diputaciones, Juntas de Agricultura, Industria y Comercio, y destacados Ingenieros de las provincias españolas más emigratorias; ya desde la primera pregunta del cuestionario, todos los interrogados en Alicante contestan que en la provincia se da emigración a Argelia, sin mencionar otra corriente (2)

Años atrás, en 1865, el Ayuntamiento de San Miguel de Salinas indicaba que en el término "no pasa un día sin ausentarse familias al extranjero, o sea, a la ciudad de Orán» (3). Como se ve, dicho consistorio identifica plenamente la emigración en general de su municipio con la emigración a Argelia, en concreto a Orán (punto de destino preferido por los emigrantes alicantinos dentro de la colonia francesa)

A comienzos del siglo $X X$ comenzó a desarrollarse con fuerza la emigra* ción hacia América. No obstante, entre el mes de julio de 1908 y el año 1913 Argelia continuaba siendo el lugar de destino preferido por los emigrantes de Elche, municipio tradicionalmente muy emigratorio: de un total de 1.879 emigrantes ilicitanos en las expresadas fechas, 1.342 marcharon a Orán y 489 a Argel (4).

(2) Vid. el interrogatorio en COMISIÓN ESPECIAL PARA ESTUDIAR LOS MEDIOS DE CONTENER EN LO POSIBLE LA EMIGRACIÓN POR MEDIO DEL DESARROLLO DEL TRABAJO, Memoria presentada al Excmo. Sr. Ministro de Fomento. Actas, Dictámenes, Interrogatorio y Documentos, Imprenta de «El Correo", Madrid, 1882, $90 \mathrm{pp}$.

(3) VERA REBOLLO. José Fernando. Tradición y cambio en el campo del Bajo Segura, Instituto de Estudios Alicantinos, Sección de Geografra, Serie $1, n{ }^{\circ}$ 93, Diputación Provincial de Alicante, Alicante, 1984. p. 55.

(4) GOZÁLVEZ PÉREZ, VICENTE, La ciudad de Elche. Estudio geográfico, Departamento de Geografía de la Universidad de Valencia, Valencia, 1976, p. 237 
Posteriormente, la Primera Guerra Mundial provocó una reducción notable del número de emigrantes a territorio argelino, al tiempo que surgieron con fuerza otros puntos de destino, donde la industria atraería a muchos emigrantes en los años del período de entreguerras: Francia, Cataluña o los centros más industrializados de la provincia de Alicante.

Por otro lado, dentro de la emigración a Argelia, los alicantinos eran, junto con los almerienses, el principal contingente de emigrantes españoles a Argelia como se aprecia en el cuadro siguiente.

\section{CUADRO ॥}

Provincias de origen de los pasajeros por mar de nacionalidad española que embarcaron con destino a Argelia desde puertos españoles entre 1885 y 1895

\begin{tabular}{|c|c|c|c|c|c|c|c|c|c|c|}
\hline \multirow[b]{2}{*}{ Años } & \multicolumn{2}{|c|}{ ALICANTE } & \multicolumn{2}{|c|}{ ALMERÍA } & \multicolumn{2}{|c|}{ MURCIA } & \multicolumn{2}{|c|}{ OTRAS } & \multicolumn{2}{|c|}{ TOTAL ESPAÑA } \\
\hline & N. ${ }^{\circ}$ & $\begin{array}{l}\% \text { det } \\
\text { total }\end{array}$ & N. ${ }^{\circ}$ & $\begin{array}{l}\% \text { del } \\
\text { total }\end{array}$ & N. ${ }^{\circ}$ & $\begin{array}{l}\% \text { del } \\
\text { total }\end{array}$ & $\mathrm{N} .{ }^{\circ}$ & $\begin{array}{l}\% \text { del } \\
\text { total }\end{array}$ & N. ${ }^{\circ}$ & $\begin{array}{c}\% \text { del } \\
\text { total }\end{array}$ \\
\hline 885 & 4.460 & 28,6 & 7.635 & 48,9 & 1.425 & 9,1 & 2.063 & 13,3 & 15.583 & 100,0 \\
\hline 1886 & 8.852 & 38,5 & 8.886 & 38,7 & 1.937 & 8,4 & 3.274 & 14,3 & 22.949 & 100,0 \\
\hline 1887 & 6.465 & 34,3 & 7.321 & 38,8 & 1.745 & 9,2 & 3.307 & 17,6 & 18.838 & 100,0 \\
\hline 1888 & 4.805 & 26,3 & 8.389 & 45,9 & 1.726 & 9,4 & 3.322 & 18,3 & 18.242 & 100,0 \\
\hline 1889 & 6.558 & 33,1 & 8.711 & 43,9 & 1.656 & 8,3 & 2.875 & 14.7 & 19 & 100,0 \\
\hline 1890 & & 34,0 & & 43,7 & 918 & 6,1 & 2.424 & 16,2 & 15 & 100,0 \\
\hline 1891 & 6.046 & 33,4 & 8.313 & 45,9 & 1.382 & 7,6 & 2.350 & 13,1 & 18 & 100,0 \\
\hline 1892 & 5.607 & 34,2 & 7.797 & 47,5 & 1.203 & 7,3 & 1.793 & 11,0 & 00 & 100,0 \\
\hline 1893 & 5.511 & 37,4 & 5.804 & 39,4 & 1.492 & 10,1 & 1.909 & 13,1 & 14.716 & 100,0 \\
\hline 1894 & 6.986 & 40,0 & 6.249 & 36,0 & 1.820 & 10,5 & 2.419 & 13,5 & 17.474 & 100,0 \\
\hline 1895 & 5.009 & 36,2 & 5.718 & 41,5 & 1.179 & 8,5 & 1.895 & 13,8 & 13.801 & 100,0 \\
\hline
\end{tabular}

FUENTE: DIRECCIÓN GENERAL DEL INSTITUTO GEOGRÁFICO Y ESTADÍSTICO, Estadística de la emigración e inmigración de España, volúmenes correspondientes a los períodos 1882-1890 y 1891-1895. Elaboración propia.

\section{Insuficiencia sistemática de fuentes documentales}

La emigración a Argelia es un capítulo de gran trascendencia en la historia demográfica reciente de nuestra provincia que, sin embargo, no ha sido estudiado con la profundidad merecida en los manuales o monografías locales.

Al margen de viejos estudios del siglo pasado y comienzos de la presente 
centuria (5), hasta los años 1970 no se acometen análisis rigurosos sobre la emigración española a Argelia. En 1974 Javier Rubio le dedicó algunas páginas en una obra suya sobre la emigración española a Francia (6). En 1975 el profesor Juan Bautista Vilar, de la Universidad de Murcia, publicó su libro Emigración española a Argelia (7). Vilar es, hoy por hoy, el mejor especialista en el tema, al que ha dedicado un número elevado de escritos, incluyendo breves estudios sobre la emigración por provincias (8). Vilar basa sus investigaciones en las fuentes francesas de los Archives d'Outre-Mer, y en fuentes españolas, fundamentalmente las estadísticas oficiales y la documentación de los Archivos Histórico Nacional y del Ministerio de Asuntos Exteriores; se trata de documentación diplomática en su mayoría.

Anteriormente, en 1968, el alicantino Antoni Seva Llinares publicó un estudio - más periodístico que geográfico - sobre los pieds-noirs y su establecimiento en la costa levantina española tras la independencia de Argelia. En su libro consideraba que unos 30.000 pieds-noirs se habrian instado en la provincia de Alicante por ser una de las provincias españolas que con anterioridad había dado mayor número de emigrantes a Argelia; en esto último centró la primera parte de su libro, aunque acudiendo normalmente a fuentes o autores franceses, donde no se distingue el origen provincial de los emigrantes españoles (9).

Existen, por tanto, estudios generales para toda España de la emigración a Argelia, y una obra centrada en los emigrantes alicantinos cuyos argumentos había que completar o rebatir con fuentes estadísticas espolas. Por ello, acometimos la realización de una investigación sobre la emigración concreta de los alicantinos a Argelia, que, a la postre, constituyó nuestra Memoria de Licenciatura (10).

Para centrarnos en el tema, geográficamente tan limitado, había que acudir a la documentación local. Había que rehuir, en lo posible, las generalizaciones de la documentación a alto nivel y acudir a las fuentes alicantinas, tanto a nivel de toda la provincia como a nivel de municipios.

(5) Vid. fundamentalmente la Memoria de la COMISIÓN ESPECIAL PARA ESTUDIAR LOS MEDIOS DE CONTENER EN LO POSIBLE LA EMIGRACIÓN..., op. cit., donde D. Carlos María Perier analiza la emigración en las provincias levantinas. Vid. también los artículos del Sr. MAZET, "Los españoles en Áfrican, Boletín de la Sociedad Geográfica de Madrid, primer semestre de 1881, pp. 388-396, y de FERNÁNDEZ FLORES, A. L., "Argelia y los españoles", Boletín de la Dirección General de Emigración, t. 1, núm. 2 y 3 (1929-1930) y t. II, núm. 1 y 2 (1930-1931).

(6) RUBiO, JAVIER, La emigración española a Francia, Horas de España, Ed. Ariel, Barcelona 1974, pp. $75-89$ y $776-189$

(7) VILAR RAMIREZ, JUAN BAUTISTA, Emigración española a Argelia, Instituto de Estudios Africanos, Madrid 1975, VIII + $537 \mathrm{pp}$.

(8) Para el caso de Alicante, vid. VILAR RAMIREZ, JUAN BAUTISTA, "Los alicantinos en la Argelia francesa (1830-1914lm. Anales de la Universidad de Alicante. Historia Conteporănea (Alicante, 1983), pp. 127-161. Se trata de la reproducción de una conferencia pronunciada por el profesor Vilar en Alicante en 1981 .

(9) SEVA LLINARES, ANTONI, Alacant. Trenta mil pieds-noirs, Col. Tresi Quatre, València, 1968, 141 pp.

(10) BONMATI ANTÓN, JOSÉ FERMÍN, La emigración de alicantinos a Argelia durante el siglo XiX y primer tercio del sigio $X X$, Memoria de Licenciatura inédita, 1986 (en prensa). 
El principal obstáculo para estudiar cualquier corriente de emigración en España durante el pasado siglo es la extremada insuficiencia de fuentes documentales. Los Ayuntamientos alicantinos, teniendo constancia de la emigración a Argelia que se producía en sus términos, apenas se interesan por su cuantificación; sólo lo hacen cuando, desde la capital de la provincia, les solicitan este tipo de información, o bien para utilizarla como exponente de su precaria situación económica. Como, por otro lado, la mayor parte de emigrados a Argelia son emigrantes temporales, es decir regresan a sus localidades tras permanecer algunos meses en territorio argelino, no tenían obligación de darse de baja en los padrones municipales, fuentes utilizadas por los funcionarios municipales para informar sobre el desarrollo de la emigración en sus respectivos términos; de ahí que, en tales informaciones, la emigración temporal no se refleja numéricamente, y sólo podrá conocerse si el funcionario, al redactar su informe, así lo hace constar.

\section{Los pasaportes}

Según la normativa vigente, para abandonar el territorio español el emigrante debía agenciarse un pasaporte. Los Ayuntamientos y el Gobierno Civil debieron llevar las relaciones de pasaportes expedidos en cada municipio (y de los pasaportes recogidos en los puertos alicantinos, al regreso de sus titulares), pero, al parecer, se han perdido en su mayoría. Los resultados obtenidos en su búsqueda en los Archivos Municipales de Alicante y Elche fueron desalentadores, y la única relación (incompleta, según parece) del Gobierno Civil o Político se reduce a los pasaportes de los emigrantes que regresaron de Argelia a través del puerto de Torrevieja entre 1855 y 1857 , conservada en el Archivo de la Diputación Provincial de Alicante (11).

Entre las fuentes inéditas, los pasaportes son los más interesantes, al recoger una serie muy completa de datos sobre el titular: nombre (a través del cual se establece la clasificación por sexos), edad, profesión, última vecindad, naturaleza y lugar de destino; informan igualmente sobre si el titular viaja sólo o acompañado (incluso, a veces, se indica también algún dato de los acompañantes), y recogen el estado civil del mismo, siendo los pasaportes una de las escasas fuentes donde se expresa tal información.

Los pasaportes son muy aprovechables sobre todo en el análisis de las profesiones de los varones por la gama muy variada de actividades que se especifica - si bien, en un alto porcentaje dice que son «jornalerosn-. En el caso de las mujeres no existe tanta variedad, pues ellas no suelen ser las titulares del documento (existían muchas restricciones legales para que las mujeres abandonasen España); cuando son las titulares, se reduce a la clasificación de "(labores) caseras" o "domesticas», o no se indica nada acerca de su profesión.

(11) ARCHIVO DE LA DIPUTACIÓN PROVINCIAL DE ALICANTE, Sección Gobierno Civil, leg. 52 


\section{Listas de embarque}

Se trata de las relaciones de pasajeros embarcados en los puertos españoles; cada lista corresponde solamente a una embarcación. En ellas, se consigna, por un lado, el lugar de destino del buque, el nombre del mismo y la naviera a la que pertenece; $y$, por otra parte, la lista de pasajeros embarcados, sus edades, su última vecindad y el número de sus billetes - debiera consignarse asimismo sus profesiones, pero siempre engloban a todos los pasajeros varones bajo el calificativo de "jornalero" y a las mujeres tras la expresión "(actividades propias de) su sexo"-.

Por lo general, no se conservan estas listas. No obstante, pudimos encontrar un corto número de las mismas referidas a servicios a Orán y Argel desde el puerto de Alicante ciudad en el Archivo de la Diputación Provincial de Alicante (12)

\section{Estadísticas oficiales de emigración}

En España estas estadísticas no recogen cifras anteriores al año 1882. A raíz de una sublevación de beréberes, en 1881, fueron asesinados por los nativos algunos trabajadores españoles en las proximidades de la ciudad de Saida, en el Oranesado. A raíz de este suceso, el Gobierno español comenzó a mostrar un interés inusitado por la emigración a Argelia de sus ciudadanos. Ni la opinión pública ni las autoridades hispanas eran favorables a la misma y el Gobierno se aprestó a combatirla. Pero, para poder combatir un fenómeno, hay que analizarlo y cuantificarlo previamente.

Para ello, en el plano estadístico se dispuso la creación de un Negociado, dependiente de la Dirección General del Instituto Geográfico y Estadístico (Ministerio de Fomento), para formar anualmente la Estadística de la emigración e inmigración de España, debiendo publicar igualmente las memorias anuales (13). Las fuentes utilizadas para conocer la emigración en cada provincia -información que los Jefes Provinciales de Estadísticas remitían a Madrid - fueron los datos proporcionados por las corporaciones locales y sobre todo por las oficinas portuarias de sanidad marítima exterior. El propio Instituto Geográfico y Estadístico consideraba que el movimiento de pasajeros por mar era, si no exacto, el método más seguro para estudiar los movimientos migratorios, pues asi lo confirmaba la observación del fenómeno tanto en España como en otros países — de hecho, era la norma que venían siguiendo otras naciones europeas para confeccionar sus respectivas estadísticas, siguiendo las propuestas del Congreso de Estadística de Bruselas, celebrado en $1853-(14)$.

(12) ARCHIVO DE LA DIPUTACIÓN PROVINCIAL DE ALICANTE, Sección Gobiemo Civil, legajos 111 (Agosto 1905), 173 (Octubre 1905), 120 (Abril 1906), 107 (Octubre 1909), 97 (noviembre 1909) y 113 (Febrero 1916).

(13) DIRECCIÓN GENERAL DEL. INSTITUTO GEOGRAFICO Y ESTADISTICO, Estadística de la emigración e inmigración de España, Memorias correspondientes a los períodus 1882-1890, 1891-1895, 1896-1900, 1901-1902, 1903-1906, 1907-1908, 1909-1911

(14) DIRECCIÓN GENERAL DEL INSTITUTO GEOGRÁFICO Y ESTADÍSTICO, Ibid, volumen correspondiente al período $1882-1890$ 
Hasta 1885, en las salidas, y 1887 , en las entradas, en estas estadísticas no se interrelacionan los datos de los pasajeros (clasificados por sexos) según su última vecindad provincial y su lugar de destino en el extranjero. Con ello, podemos conocer el número de emigrantes alicantinos a Argelia, distinguidos por sexos, aunque nada se indica acerca de la edad y profesión de los mismos.

Con el tiempo, en las estadísticas del Instituto Geográfico y Estadístico se va perdiendo información. Se sigue clasificando a los pasajeros que marchan o regresan desde el extranjero a través de puertos españoles por sexo, edad, profesión y nacionalidad, pero, desde 1896, no se indica los lugares de destino de los pasajeros alicantinos $y$, desde 1899, ni siquiera se distingue la última vecindad provincial de los pasajeros. Este último dato reaparece (sólo en las salidas) en 1911, aunque desde esa fecha no vuelve a indicarse los lugares de destino de los pasajeros de cada provincia española.

En 1912 las estadísticas oficiales pasan a denominarse Estadística del movimiento de buques y pasajeros por mar con el exterior (15). La primera parte de este nuevo tipo de estadísticas consiste en un breve informe y comentario de las cifras absolutas de pasajeros ( $y$ buques) por mar, clasificados por lugares de procedencia o destino, sexo, edad, profesión, nacionalidad y mes de entrada o salida de España. En la segunda parte, se publican los resúmenes numéricos anuales siguiendo los mismos criterios y clasificaciones que en el informe previo. Desafortunadamente, en dichas clasificaciones no se distingue a los pasajeros según su última vecindad provincial. Existe un apartado especial en que se clasifica a los pasajeros por provincias de última vecindad (sólo para las salidas), pero sin distinguir los puntos de destino en el extranjero de los mismos.

\section{Fichas mensuales del movimiento de buques $y$ pasajeros en los puertos de la provincia de Alicante.}

Estas fichas, conservadas en el Archivo de la Junta Provincial del Instituto Nacional de Estadística en Alicante (16), son la base de la información de cada Delegación Provincial de Estadística enviaba al Instituto Geográfico y Estadístico de Madrid para la elaboración de las estadísticas oficiales de emigración e inmigración, que acabamos e estudiar; por tanto, los criterios seguidos en las clasificaciones de los pasajeros eran idénticos. Como ya se dijo, se basan en datos proporcionados por las oficinas de sanidad marítima exterior.

\footnotetext{
(15) SUBSECRETARÍA DE TRABAJO Y ACCIÓN SOCIAL DEL MINISTERIO DE TRABAJO. SUBDIRECCIÓN GENERAL DE ESTADISTICA PÚBLICA. Estadística del movimiento de buques y pasajeros por mar con el exterior, distintos volúmenes correspondientes a los perfodos 1912-1913, 1914, 1915, 1916, 19171918,1919,1920-1922,1923-1925,1926-1929,1930,1931 y 1932-1934. La misma estadística, para el período 1935-1944, fue publicada por el INSTITUTO NACIONAL DE ESTADISTICA.

(16) ARCHIVO DE LA DELEGACIÓN PROVINCIAL DEL INSTITUTO NACIONAL DE ESTADISTICA EN ALICANTE. Movimiento de pasajeros por mar con el exterior en los puertos de la provincia de Alicante. En concreto, se conservan las fichas mensuales de los años 1882 a 1888 (respectivamente los legajos $16,17,18,24,23,29$ y 30 ), 1893-1896 (respectivamente los legajos $38,40,42$ y 46 ) y $1902-1935$ (legajos $77,94,113,121,128,137,144,155,167,175,194,210,221$ y 241 ).
} 
En esta fuente inédita podemos distinguir los puntos destino de los pasajeros que embarcan en los puertos alicantinos (también los puntos de procedencia en el extranjerol, entre ellos Argelia. Ello supone una mayor precisión informativa respecto a las estadísticas oficiales publicadas, donde, en el caso de los puertos alicantinos (y españoles, en general), no se especifica los puntos extranjeros de procedencia o destino del pasaje, y en el caso del movimiento maritimo con Argelia, no se distingue dicho movimiento por puertos españoles.

Con estas fichas, no podemos conocer el número exacto de pasajeros alicantinos que viajan a/desde Argelia a través de los puertos alicantinos, al no interrelacionar los datos sobre última vecindad provincial y lugar extranjero de destino del pasaje; pero son las estadísticas más fiables a la hora de acudir a métodos indirectos para el análisis de la corriente de emigración de los alicantinos a Argelia, pues la mayor parte de los emigrantes de nuestra provincia utilizaba estos puertos para desplazarse o regresar desde territorio argelino.

\section{Conclusiones}

Existen otras fuentes locales complementarias (prensa alicantina de la época, Boletines Oficiales de la Provincia de Alicante, Encuestas Municipales sobre Cambios de Residencia Legal en los términos de nuestra provincia, Estadísticas sobre el Movimiento Social de la Población de la Provincia de Alicantel, pero, en definitiva, el hecho más destacable sobre las fuentes para el estudio de cualquier corriente de emigración en España para la época considerada es precisamente la insuficiencia de las mismas. Prácticamente, se reducen a estadísticas basadas en datos portuarios, con las cuales es de todo punto imposible conocer la cifra exacta de emigrantes españoles en general $y$ alicantinos en concreto, porque:

1. ${ }^{\circ}$ En estas estadísticas no se recoge el número de emigrantes que se trasladan a Argelia desde territorios no españoles.

2. ${ }^{\circ}$ Tampoco recogen obviamente la emigración clandestina, hecho tantas veces deplorado por las autoridades y que, según parece, supuso en ocasiones cifras muy elevadas de emigrantes. yoría.

3. ${ }^{\circ}$ No todos los pasajeros por mar serían emigrantes, aunque sí la ma-

4. ${ }^{\circ}$ Las listas portuarias que sirven de base para la formación de las estadísticas solían ser más precisas en lo referente a las entradas que en las salidas, fundamentalmente porque, por razones de indole sanitaria, las autoridades portuarias extremaban más su celo en el control de las entradas.

Por todo ello, no podemos pretender otra cosa con estas fuentes que no sea señalar las tendencias de la emigración, tanto en cifras absolutas como 
relativas. Muchas veces, el investigador debe acudir a métodos indirectos que, generalmente, consisten en analizar las cifras totales de pasajeros embarcados por los puertos de la provincia de Alicante con destino a Argelia (o procedentes de este territorio). Naturalmente no todos estos pasajeros eran alicantinos, ni todos los alicantinos marcharian a Argelia desde los puertos de nuestra provincia. Pero concurren dos circunstancias que permiten considerar este método como el más válido de cuantos pudiera ensayarse para estudiar la emigración de los alicantinos al territorio argelino:

- En los años en que estadísticamente se ha podido comprobar, más del $75 \%$ de los alicantinos que entraron o salieron por mar al extranjero desde puertos españoles lo hicieron por los de la provincia de Alicante.

- La práctica totalidad (casi siempre más del 90\%) de los pasajeros que salieron/entraron a/desde el extranjero a través de los puertos de la provincia de Alicante marcharon/procedieron a/de Argelia. 